\title{
Real options games analysis of sleeping patents
}

\author{
Chi Man Leung and Yue Kuen Kwok ${ }^{1}$ \\ Department of Mathematics, Hong Kong University of Science and \\ Technology, Hong Kong
}

\begin{abstract}
Using the real options game approach, we analyze the two-stage preemptive patent-investment race between an incumbent and a challenger (new entrant) in a product market with profit flow uncertainty. The challenger can gain entry into the monopolized product market dominated by the incumbent by patenting related technologies for a substitute product. To maintain its monopolized advantage, the incumbent has an incentive to block challenger's entry by patenting the substitute product before the challenger. Either firm can pay an upfront fee to gain an immediate acquisition of the patent, and subsequently holding the real option to develop the new substitute product. We provide a full characterization of the optimal strategies adopted by the incumbent and challenger firm in this asymmetric patent-investment race. In particular, we examine the phenomena of sleeping patent, where the patented product is not launched immediately into the product market.
\end{abstract}

Keywords: patent races, sleeping patents, real options games.

\section{Introduction}

This paper considers a real options game model that analyzes the two-stage preemptive patent-investment race between an incumbent firm and a challenger in a product market under asymmetric costs and profit flow rates. The product market with profit flow uncertainty is currently monopolized by an incumbent firm. The entry of a challenger (new entrant) into the product market can be gained by patenting and developing a substitute product. On the other hand, the incumbent can block the challenger's entry by acquiring the patent of the substitute product and maintain its monopoly status. For the challenger, the patent is an entry ticket into the product market.

\footnotetext{
${ }^{1}$ Correspondence author; email: maykwok@ust.hk
} 
The real option right to launch the substitute product and earn a positive profit flow is won by the first firm which obtains the patent. In this paper, a continuous time real investment option model is developed to investigate the strategic interaction between the incumbent and challenger under an asymmetric patent-investment race.

The real options approach accommodates market uncertainty, irreversibility of investment and ability to delay entry in investment decisions. It is called the real option since it exploits the analogy between a financial call option and the firm's investment opportunity (Dixit and Pindyck, 1994). Pakes (1986) initiates the use of real options to analyze the option value of holding European patent stocks. He estimates the distribution of the returns earned from holding patents by solving the associated implied optimal stopping model. Reiss (1998) applies real options valuation to determine whether and when a firm should patent and adopt an innovation if the arrival time of a competitor is stochastic. He analyzes how competition and patent fee level affect the various strategies on patenting and investing, and the maximum fixed R\&D expenditures. Weeds (1999) applies a real options framework with both economic and technological uncertainty to show that a patent that is left unexploited may be resulted from forward looking behavior when there is no anticompetitive motive of the firm holding the patent. Some possible measures that are taken to enforce the development of sleeping patents may harm incentives for firms to engage in research. Takalo and Kanniainen (2000) challenge the widely held belief that patents always speed up technological development. Using real options analysis, they show that patent protection raises the threshold value of market introduction and enhances the ability of the innovator to wait. A thorough review of research on economic valuation of patents under real options framework can be found in Sereno (2008).

In our two-stage real options game model, the incumbent and challenger both hold the real option to invest in a two-stage sequential patent-investment project. In the first stage, the two firms compete to buy the patent for a fixed cost common to both firms. Once the patent is acquired, the patent holder has the real option to commercialize the patented product in the second stage with a commercialization cost. When the substitute product is developed by the incumbent, the firm earns an enhanced profit flow rate through operating the two products. On the other hand, suppose the challenger develops the substitute product, the incumbent loses its monopoly thus resulting in a lower profit flow rate when the product market is operated in duopoly. Asymme- 
try in the commercialization cost of the substitute product is assumed. We analyze the optimal timing of the two sequential investment decisions: acquisition of the patent and commercialization of the substitute product. The uncertainty in the profit flow rate is subject to stochastic shock as modeled by a stochastic state variable. The patent can be considered as a contingent claim on the commercialization value of the patented asset, so our two-stage patent-investment model resembles a compound option model. Our patentinvestment race model exploits the investment policies associated with the real option value of patents. For the first stage, either firm chooses to acquire the patent optimally when the stochastic state variable reaches certain threshold, called the patent trigger. For the incumbent, the optimal patent acquisition decision represents a tradeoff between the benefit of holding the real option of developing the substitute product and the loss in profit flow rate resulting from being preempted. In other words, the incumbent chooses to preempt its rival if the patent cost is less than the total benefits that are gained by preventing challenger's entry. Under certain market conditions, the act of preemptive patenting may lead to sleeping patent. That is, the patented substitute product stays unexploited for a period of time, a decision on delay of commercialization decided optimally by the holder of the patent.

Recent real options research has also been directed to the analysis of strategic investment decisions under the real options game framework (Grenadier, 1996; Kong and Kwok, 2007). The issues of patent races, sleeping patents and the persistence of monopoly have been studied in several earlier papers (Gilbert and Newbery, 1982; Harris and Vickers, 1985). Lambrecht (2000) initiates the analysis of sleeping patent in patent-investment races but his framework is limited to symmetric firms. In another related work, Hsu and Lambrecht (2007) considers the one-stage patent race models under asymmetric information where the challenger has complete information about the incumbent whereas the incumbent does not know the exact investment cost of its competitor. Their one-stage model takes the commercialization cost of the product to be zero, so sleeping patent does not occur. Our model combines the two-stage real options game of patenting and commercialization, asymmetry in status, cost and profit flow rate. In our framework of analysis, we quantify the incentive for preemptive patenting (first mover advantage) adopted by the two competing firms. We examine how asymmetry in status, cost and profit flow rate would affect the optimal investment strategies adopted by the incumbent and challenger. We also analyze how these real option values may be undermined by the fear of preemption. In particular, 
we explore the conditions on the relative magnitude of costs and profit flow rates under which sleeping patent may occur.

This paper is organized as follows. In the next section, we present the model formulation of the two-stage asymmetric patent-investment race between the incumbent and entrant firm. The first stage is concerned with the acquisition of the patent while the second stage is the commercialization of the patented product. We compute the value functions of the two firms and consider the various types of trigger strategies of the incumbent and its challenger. In particular, we determine the Marshallian triggers of the two firms. In Section 3, we explore the various forms of preemptive strategies adopted by both the incumbent and challenger and identify which firm is more efficient with regard to taking the preemptive action ahead of its rival. We then examine the conditions under which sleeping patent may occur. In Section 4, we examine how the optimal strategies of patenting and commercialization depend on the various cost and profit flow rate parameters. We provide the full characterization of the preemptive strategies and sleeping patent phenomena with respect to the relative magnitude of profit flow rates and costs. Concluding remarks are presented in the last section.

\section{Real options game model of patent-investment race}

We consider the two-stage patent-investment race between an incumbent (Firm $i$ ) and its challenger as potential new entrant (Firm e). In our twofirm real options game model, the strategic competition among the two firms is characterized by the firm's own assessment of the profit flow rate and the potential preemptive threat. The threat of preemption would reduce the flexibility of delay and option value of waiting. The more efficient firm may epsilon preempt its rival and it is still able to preserve some option value of waiting. The incumbent is now serving a monopolized market with a product. The stochastic profit flow rate received by Firm $i$ as generated from the monopolized product is given by the Geometric Brownian process $x_{t}$. The profit flow rate $x_{t}$ is governed by the following stochastic differential equation:

$$
d x_{t}=\mu x_{t} d t+\sigma x_{t} d Z_{t}
$$


Here, $\mu$ is the constant risk-adjusted drift rate, $\sigma$ is the constant volatility and $Z_{t}$ is the standard Brownian process. Since the product market may not behave like the capital market, the usual preference free option valuation approach may not be applicable when valuing real options. In our model, the discount rate adopted by the two firms is taken to be the riskless interest rate $r$. This can be justified by assuming either (i) firms are risk neutral investors which take zero market price of risk, or (ii) the uncertainty over the profit flow rate can be spanned by existing assets in the financial market. As usual, we assume the no-bubble condition where $\mu<r .{ }^{2}$ The present value of the perpetual profit flow stream $x_{t}$ is given by

$$
E_{Q}^{t, x}\left[\int_{t}^{\infty} e^{-r(u-t)} x_{u} d u\right]=\frac{x}{r-\mu},
$$

where $E_{Q}^{t, x}$ denotes the expectation under the risk neutral measure $Q$ conditional on the information at time $t$, with $x_{t}=x$.

We assume that the new entrant's entry into the product market can only be gained by patenting related technologies for a substitute product. On the other hand, the incumbent may block the entry of the entrant by acquiring the patent of the substitute product prior to its competitor. It is assumed that either firm can pay an upfront fee $C$ to gain an immediate acquisition of the patent, and the patent is assumed to be of infinite life. The patent holder has the privilege to block entry of its competitor in the exploitation of the substitute product. In addition, he holds the real option to commercialize the substitute product. It may occur that he just lets the patent sleep, that is, the competitor's entry is blocked but the substitute product is not launched immediately. After the first stage of acquiring the patent, a sunk cost of investment is required to commercialize the substitute product in the second stage. We let $K_{j}, j=i, e$, denote the respective commercialization cost required for the incumbent and challenger. When both products are operated by the incumbent, the stochastic profit flow rate is increased to $\left(1+\pi_{i}^{+}\right) x_{t}$, where $\pi_{i}^{+}>0$. Otherwise, suppose the challenger (Firm $e$ ) acquires the patent and later commercializes the substitute product, the stochastic profit flow rate received by Firm $e$ is $\pi_{e} x_{t}$. In this case, the market

\footnotetext{
${ }^{2}$ Write $\delta=r-\mu$, and $\delta>0$ when $\mu<r$. Here, $\delta$ can be interpreted as the opportunity cost of delaying the act of patenting (similar to the dividend yield in an American call option model). If $\delta$ is non-positive, there is no opportunity cost of keeping the real option alive. The firms will never exercise the real option, no matter how high is the profit flow rate.
} 
becomes duopoly with two products. As a consequence, the incumbent's profit flow rate is then reduced to $\left(1-\pi_{i}^{-}\right) x_{t}$, where $0<\pi_{i}^{-}<1$.

Since the profit flow state variable is assumed to be Markovian, the firms would adopt a single trigger strategy where they choose to acquire the patent of the substitute product when the state variable $x_{t}$ reaches certain trigger threshold from below. How do we quantify the incentive for preemptive patenting adopted by the two firms to achieve the corresponding first mover advantage? For the incumbent, by paying the upfront patent $\operatorname{cost} C$ and the subsequent sunk cost of investment $K_{i}$, it achieves an increase in profit flow rate of $\pi_{i}^{+} x_{t}$ when it monopolizes the market with two products. We expect that the incumbent chooses to preempt if the patent cost $C$ is less than the expected value of the profits gained by holding the investment option of the substitute product and preventing the loss in profit flow rate due to new entrant's entry. On the other hand, the challenger has the real option of acquiring the patent at the patent $\operatorname{cost} C$ and later launching the substitute product at the sunk cost $K_{e}$ to earn the profit flow rate of $\pi_{e} x_{t}$. However, the economic value of this real option may be undermined by the preemptive patenting of the incumbent. This is because the entrant may be forced to exercise the real option at a lower trigger threshold to achieve strategic preemption of the competitor.

First, we would like to determine the patent and commercialization triggers, and the Marshallian triggers of the two firms. Here, the Marshallian trigger of a firm is defined to be the breakeven trigger level at which the patent cost balances the real option value of acquiring the patent. We also compute the value functions of the two firms in the two stages. The first stage corresponds to prior acquisition of the patent while in the second stage the patent has been acquired by one of the two firms.

\subsection{Trigger thresholds and value function of the en- trant}

It is relatively easier to determine the trigger thresholds and the value function of the entrant since it does not operate any product prior to the patenting and commercialization of the substitute product. First, we find the optimal non-competitive patent trigger and commercialization trigger under no preemptive threat from the incumbent. Without strategic interaction with a competing firm, we show that the product will be commercialized imme- 
diately once the patent is acquired. However, under potential preemptive threat of the competing firm, the entrant firm may choose to acquire the patent at a trigger level of $x_{t}$ that is lower than the non-competitive patent trigger. By striking the balance between the real option value of holding the patent and the patent cost, we determine the Marshallian trigger below which the entrant firm will never acquire the patent.

Assuming no preemptive threat from the incumbent (Firm $i$ ), we would like to determine the non-competitive patent trigger $x_{e p}^{*}$ at which it is optimal for the entrant (Firm $e$ ) to acquire the patent with patent $\operatorname{cost} C$ and the commercialization trigger $x_{e c}^{*}$ at which it is optimal for the entrant to commercialize the substitute product at the known sunk cost $K_{e}$. Let $V_{e}^{(1)}(x)$ and $V_{e}^{(2)}(x)$ be the value function of Firm $e$ in the first stage of patenting and the second stage of commercialization, respectively, when the stochastic state variable $x_{t}$ assumes the value $x$. As in usual two-stage investment models, we solve for the value functions backward starting from stage two then to stage one. When $x \geq x_{e c}^{*}$, the patented product will be commercialized and the present expected value of the perpetual profit flow stream is equal to $\frac{\pi_{e} x}{r-\mu}$. Hence, it can be seen that

$$
V_{e}^{(2)}(x)=\frac{\pi_{e} x}{r-\mu}-K_{e} \quad \text { for } x \geq x_{e c}^{*} .
$$

When $x<x_{e c}^{*}$, Firm $e$ holds the real option value of commercialization of the substitute product through holding the patent. Following a similar derivation as in Lambrecht (2000), this real option value is given by

$$
V_{e}^{(2)}(x)=\left(\frac{\pi_{e} x_{e c}^{*}}{r-\mu}-K_{e}\right)\left(\frac{x}{x_{e c}^{*}}\right)^{\beta} \quad \text { for } x<x_{e c}^{*}
$$

where

$$
x_{e c}^{*}=\frac{\beta}{\beta-1} \frac{K_{e}(r-\mu)}{\pi_{e}} \text { and } \beta=\frac{-\left(\mu-\frac{\sigma^{2}}{2}\right)+\sqrt{\left(\mu-\frac{\sigma^{2}}{2}\right)^{2}+2 r \sigma^{2}}}{\sigma^{2}} \text {. }
$$

It can be shown that $\beta>1$ when $\mu<r$. The factor $\left(\frac{x}{x_{e c}^{*}}\right)^{\beta}$ represents the present value of a contingent claim that pays $\$ 1$ when the stochastic state variable evolves from the current value $x$ and later reaches a higher value $x_{e c}^{*}$. Using the above result for $x_{e c}^{*}$, the real option value can be simplified as

$$
\left(\frac{\pi_{e} x_{e c}^{*}}{r-\mu}-K_{e}\right)\left(\frac{x}{x_{e c}^{*}}\right)^{\beta}=\frac{K_{e}}{\beta-1}\left(\frac{x}{x_{e c}^{*}}\right)^{\beta} .
$$


Combining all these results together, we obtain

$$
V_{e}^{(2)}(x)=\left\{\begin{array}{ll}
\frac{K_{e}}{\beta-1}\left(\frac{x}{x_{e c}^{*}}\right)^{\beta} & \text { for } x<x_{e c}^{*} \\
\frac{\pi_{e} x}{r-\mu}-K_{e} & \text { for } x \geq x_{e c}^{*}
\end{array} .\right.
$$

Similarly, we can easily deduce the entrant's value function in the first stage by observing that the payoff upon acquisition of the patent is now given by $V_{e}^{(2)}(x)-C$. One then obtains

$$
V_{e}^{(1)}(x)= \begin{cases}{\left[V_{e}^{(2)}\left(x_{e p}^{*}\right)-C\right]\left(\frac{x}{x_{e p}^{*}}\right)^{\beta}} & \text { for } x<x_{e p}^{*} \\ V_{e}^{(2)}(x)-C & \text { for } x \geq x_{e p}^{*}\end{cases}
$$

where

$$
x_{e p}^{*}=\frac{\beta}{\beta-1} \frac{\left(C+K_{e}\right)(r-\mu)}{\pi_{e}} .
$$

Since $C>0$, so we have $x_{e p}^{*}>x_{e c}^{*}$. This result indicates that once Firm $e$ purchases the patent when $x_{t}$ reaches the patent trigger $x_{e p}^{*}$, it should commercialize the product immediately as an optimal strategy (Lambrecht, 2000). Under no preemptive threat from its competitor, the entrant firm will not let the patent stay unexploited. In fact, acquiring the patent too soon means paying a higher time value of the patent cost $C$, so it is a nonoptimal strategy. However, when there exists potential preemptive threat from the incumbent, Firm $e$ may choose to acquire the patent at a lower trigger threshold of $x_{t}$ so as to avoid being preempted and leading to complete loss of the real option value of patenting.

Under the strategic patent-investment race between the incumbent and entrant, each firm faces the threat of preemption by its competitor. When one of the two firms acquires the patent, the other firm is blocked from entry to the product market. The firm that is preempted then has zero real option value of patenting. Due to the threat of being preempted, Firm $e$ may choose to acquire the patent in the first stage at some strategic preemption trigger $x_{e s}^{*}$ that may be below the non-competitive optimal patent trigger $x_{e p}^{*}$. We would like to characterize the range of $x_{e s}^{*}$. Obviously, $x_{e p}^{*}$ represents the upper bound of $x_{e s}^{*}$. What would be the lower bound of $x_{e s}^{*}$ ?

When Firm $e$ chooses to preempt its competitor by purchasing the patent at the strategic preemption trigger $x_{e s}^{*}$, this occurs only when $V_{e}^{(2)}(x) \geq C$. We define the Firm $e$ 's preemption value function by

$$
\Phi_{e}(x)=V_{e}^{(2)}(x)-C,
$$


which represents the strength of preemption of Firm $e$. It can be shown that

$$
\Phi_{e}\left(x_{e p}^{*}\right)=V_{e}^{(2)}\left(x_{e p}^{*}\right)>0 \text { and } \Phi_{e}(0)=-C<0 .
$$

Since $\Phi_{e}(x)$ is continuous and monotonically increasing in $x$, there exists an unique root $\bar{x}$ such that $\Phi(\bar{x})=0$. Firm $e$ will preempt only when $x \geq \bar{x}$ giving a non-negative value of the preemption value function $\Phi_{e}(x)$, and will not choose to preempt otherwise. We denote this break-even trigger level $\bar{x}$ to be $x_{e m}^{*}$ and it represents the lower bound of $x_{e s}^{*}$. Following Hsu and Lambrecht (2007), this lower bound of $x_{e s}^{*}$ is called the Marshallian trigger of Firm $e^{3}$. Therefore, $x_{e s}^{*}$ is seen to lie within the interval $\left[x_{e m}^{*}, x_{e p}^{*}\right]$. The determination of the exact value of $x_{e s}^{*}$ requires the examination of the strategic interaction between the two competing firms, the details of which will be discussed in Section 3 .

Recall that $x_{e m}^{*}$ satisfies

$$
\Phi_{e}\left(x_{e m}^{*}\right)=V_{e}^{(2)}\left(x_{e m}^{*}\right)-C=0 .
$$

From Eq. (2.3), $V_{e}^{(2)}(x)$ has different functional forms over different intervals, depending on $\Phi_{e}\left(x_{e c}^{*}\right)>0$ or otherwise. It can be shown that

$$
\Phi_{e}\left(x_{e c}^{*}\right)>0 \Leftrightarrow K_{e}>C(\beta-1),
$$

and correspondingly,

$$
x_{e m}^{*}=\left\{\begin{array}{ll}
\frac{\left(C+K_{e}\right)(r-\mu)}{\pi_{e}} & \text { for } K_{e} \leq C(\beta-1) \\
\frac{\beta}{\beta-1} \frac{K_{e}(r-\mu)}{\pi_{e}}\left[\frac{C(\beta-1)}{K_{e}}\right]^{\frac{1}{\beta}} & \text { for } K_{e}>C(\beta-1)
\end{array} .\right.
$$

\subsection{Trigger thresholds and value function of the in- cumbent}

Like the entrant firm, the incumbent also possesses the real option of patenting that blocks the entry of the competitor. The patent entitles its holder the

\footnotetext{
${ }^{3}$ The Marshallian trigger usually refers to the trigger threshold of entering into an investment project based on the net present value rule, corresponding to the case of zero volatility in real option of investment. The Marshallian trigger thus represents the trigger threshold at which the investor breaks even. This break-even trigger would be the lower bound of the investment trigger.
} 
real option value of commercialization of the new product. Let $V_{i}^{(2)}(x)$ denote the incumbent's value function of this real option of commercialization and $x_{i c}^{*}$ denote the corresponding commercialization trigger of the incumbent (Firm $i$ ). The second stage value function and the commercialization trigger of the incumbent are found to be

$$
V_{i}^{(2)}(x)=\left\{\begin{array}{ll}
\frac{K_{i}}{\beta-1}\left(\frac{x}{x_{i c}^{*}}\right)^{\beta} & \text { for } x<x_{i c}^{*} \\
\frac{\pi_{i}^{+} x}{r-\mu}-K_{i} & \text { for } x \geq x_{i c}^{*}
\end{array},\right.
$$

where

$$
x_{i c}^{*}=\frac{\beta}{\beta-1} \frac{K_{i}(r-\mu)}{\pi_{i}^{+}} .
$$

On the other hand, the preemptive patenting of Firm $e$ causes potential decrease of the profit flow rate of Firm $i$ due to the loss of monopoly position held by Firm $i$. Let $L_{i}(x)$ be the value function of this loss of monopoly associated with the decrease of Firm $i$ 's profit flow rate of amount $\pi_{i}^{-} x_{t}$ when the entrant exercises preemptive patenting and subsequently commercializes the product. Once the patent has been acquired by Firm $e$, the expected value of the loss of Firm $i$ 's profit flow stream is $\frac{\pi_{i}^{-} x}{r-\mu}$ when Firm $e$ has commercialized the product, where $x \geq x_{e c}^{*}$. On the other hand, since the expected loss value is $\frac{\pi_{i}^{-} x_{e c}^{*}}{r-\mu}$ when $x_{t}$ reaches the Firm $e$ 's commercialization trigger $x_{e c}^{*}$, so the expected loss value when $x_{t}$ stays below $x_{e c}^{*}$ is given by $\frac{\pi_{i}^{-} x_{e c}^{*}}{r-\mu}\left(\frac{x}{x_{e c}^{*}}\right)^{\beta}$. In summary, this loss function (negative valued) is given by

$$
L_{i}(x)=\left\{\begin{array}{ll}
-\frac{\pi_{i}^{-} x_{e c}^{*}}{r-\mu}\left(\frac{x}{x_{e c}^{*}}\right)^{\beta} & \text { for } x<x_{e c}^{*} \\
-\frac{\pi_{i}^{-} x}{r-\mu} & \text { for } x \geq x_{e c}^{*}
\end{array} .\right.
$$

Similar to the earlier calculation performed for the entrant's triggers, we would like to determine the upper bound and lower bound of the strategic preemption trigger $x_{i s}^{*}$ of Firm $i$. The upper bound is naturally the noncompetitive patent trigger $x_{i p}^{*}$ that is given by

$$
x_{i p}^{*}=\frac{\beta}{\beta-1} \frac{\left(C+K_{i}\right)(r-\mu)}{\pi_{i}^{+}} .
$$

Firm $i$ may choose to delay patenting until $x_{t}$ reaches $x_{i p}^{*}$ from below when there is no threat of preemption from its competitor. By paying the upfront fee $C$ of acquiring the patent, Firm $i$ avoids the potential reduction of 
the profit flow stream. Instead it receives the real option of commercialization that enhances the profit flow rate by operating the two products. The preemption function of Firm $i$ is appropriately defined by

$$
\Phi_{i}(x)=V_{i}^{(2)}(x)-C-L_{i}(x),
$$

and the unique solution to $\Phi_{i}(x)=0$ is defined as the Marshallian trigger $x_{i m}^{*}$ of Firm $i$. Again, Firm $i$ never chooses to exercise when $x_{t}$ falls below $x_{i m}^{*}$ since $\Phi_{i}(x)<0$ when $x<x_{i m}^{*}$. Therefore, $x_{i m}^{*}$ represents the lower bound of $x_{i s}^{*}$. Unlike $x_{e m}^{*}$, we are unable to find an explicit formula for $x_{i m}^{*}$.

\section{Strategic interaction between the incum- bent and entrant}

In the last section, we have obtained the non-competitive patent triggers and commercialization triggers of the incumbent and entrant. We have also derived the Marshallian trigger of each firm below which the firm never exercises the acquisition of the patent since this would lead to negative payoff to the firm. The firm which has the lower Marshallian trigger is considered to be more economically efficient in patenting the substitute product. The more efficient firm chooses to preempt the less efficient firm by acquiring the patent just before the less efficient firm becomes indifferent between patenting and being preempted. This strategy is called $\varepsilon$-preemption. Once we have obtained the various triggers $x_{j c}^{*}, x_{j p}^{*}$ and $x_{j m}^{*}$, where $j=i, e$, for both firms, we would like to deduce the strategic preemption triggers $x_{j s}^{*}, j=i, e$. Also, we would like to deduce the necessary and sufficient condition for the occurrence of sleeping patent, where the firm chooses to delay the commercialization of the patent after the acquisition of the patent. To simplify our analysis, we make the assumption that the current value of the stochastic state variable $x_{t}$ is below the minimum of the two Marshallian triggers.

\section{Preemption strategy of the efficient firm}

Recall that the strategic preemption trigger $x_{j s}^{*}$ always lie within the interval $\left[x_{j m}^{*}, x_{j p}^{*}\right], j=i, e$. When the Marshallian trigger of one firm is higher than that of its competitor, the more efficient competitor would exercise preemptive patenting either at a trigger threshold that is slightly below the firm's Marshallian trigger (this is termed $\varepsilon$-preemption) or at the competitor's non- 
competitive patent trigger. Strictly speaking, the strategic preemption trigger is defined only for the efficient firm, which is considered to be the winner of this patent race. We use the labels " $w$ " and "l" to denote the winner firm and loser firm, respectively. Assuming $x_{i m}^{*}$ and $x_{e m}^{*}$ to be $\operatorname{distinct}^{4}$, we set

$$
x_{w m}^{*}=\min \left\{x_{i m}^{*}, x_{e m}^{*}\right\} \text { and } x_{l m}^{*}=\max \left\{x_{i m}^{*}, x_{e m}^{*}\right\} .
$$

The strategic preemption strategy of the winner firm depends on the relative magnitude of $x_{l m}^{*}$ and $x_{w p}^{*}$. More specifically:

(i) When $x_{l m}^{*} \leq x_{w p}^{*}$, the winner firm exercises the option of acquiring the patent at $x_{l m}$ by $\varepsilon$-preemption. In this case, we have $x_{w s}^{*}=x_{l m}^{*}-\varepsilon$, where $\varepsilon>0$ is an arbitrarily small constant.

(ii) When $x_{l m}^{*}>x_{w p}^{*}$, the winner firm exercises the option of patenting at the optimal patent trigger $x_{w p}^{*}$ without the preemptive threat from its less efficient competitor. In this case, we have $x_{w s}^{*}=x_{w p}^{*}$.

In summary, the strategic preemption trigger $x_{w s}^{*}$ of the winner firm is given by

$$
x_{w s}^{*}=\max \left\{x_{w m}^{*}, \min \left\{x_{w p}^{*}, x_{l m}^{*}-\varepsilon\right\}\right\} .
$$

\section{Sleeping patent}

As explained earlier, when there is no preemptive threat from the competing firm, sleeping patent never occurs since $x_{j c}^{*}<x_{j p}^{*}, j=i, e$. However, when the more efficient firm is under preemptive threat from its competitor, it may occur that the strategic preemption trigger $x_{w s}^{*}$ of the winner firm is below its commercialization trigger $x_{w c}^{*}$. Recall that the winner firm chooses to $\varepsilon$-preempt its rival when $x_{e m}^{*}<x_{w p}^{*}$, and under such scenario, $x_{w s}^{*}$ is set to be $x_{l m}^{*}$. Sleeping patent occurs when the winner firm exercises preemptive patenting at $x_{l m}^{*}-\varepsilon$ and commercializes the product when the higher threshold $x_{w c}^{*}$ is reached. Together with the properties that

$$
x_{w m}^{*}<x_{l m}^{*} \text { and } x_{w c}^{*}<x_{w p}^{*},
$$

we deduce that sleeping patent occurs if and only if

$$
x_{w m}^{*}<x_{l m}^{*} \leq x_{w c}^{*}<x_{w p}^{*} .
$$

\footnotetext{
${ }^{4}$ When $x_{i m}^{*}=x_{e m}^{*}$, both firms would rush to preempt its opponent at the common Marshallian threshold. The chance of winning the patent race is $50 \%$ for either firm.
} 
In the unlikely event that $x_{l m}^{*}=x_{w c}^{*}$, we have $x_{w s}^{*}=x_{l m}^{*}-\varepsilon$ so that the product is almost commercialized immediately in the next moment right after preemptive patenting.

The information about the relative magnitude of $x_{l c}^{*}$ and $x_{l p}^{*}$ of the less efficient firm is immaterial in the analysis of "sleeping patent". Since we always have

$$
x_{w m}^{*}<x_{l m}^{*}, \quad x_{w c}^{*}<x_{w p}^{*}, \quad \Phi_{w}\left(x_{w m}^{*}\right)=0 \quad \text { and } \quad \Phi_{l}\left(x_{l m}^{*}\right)=0,
$$

condition (3.3) holds if and only if

$$
\Phi_{e}\left(x_{l m}^{*}\right)>0 \text { and } \Phi_{l}\left(x_{w c}^{*}\right)>0 .
$$

The procedure for checking whether the "sleeping patent" phenomenon occurs can be summarized as follows:

1. We determine whether Firm $e$ or Firm $i$ represents the winner firm by comparing $x_{e m}^{*}$ [see Eq. 2.6] and $x_{i m}^{*}$ [see Eq. 2.11]. The firm with the lower Marshallian threshold is the winner firm.

2. Once the winner firm has been identified, we determine whether condition (3.3) holds.

\section{Summary of strategic preemptive patenting of the efficient firm}

1. When $\Phi_{w}\left(x_{w c}^{*}\right) \leq 0$, which is equivalent to $x_{w c}^{*} \leq x_{w m}^{*}$, "sleeping patent" would never occur. This is because $x_{w c}^{*} \leq x_{w m}^{*}<x_{w s}^{*}$. Now, the strategic preemptive patenting trigger of the efficient firm is given by $x_{w s}^{*}=\min \left(x_{l m}^{*}, x_{w p}^{*}\right)$. The new substitute product is commercialized immediately once the patent is acquired.

2. As a result, $\Phi_{w}\left(x_{w c}^{*}\right)>0$ is a necessary condition for the occurrence of "sleeping patent". When the condition $\Phi_{w}\left(x_{w c}^{*}\right)>0$ is satisfied, there are 3 possible scenarios. Recall that we always have $x_{w m}^{*}<x_{w c}^{*}<x_{w p}^{*}$, and by definition, $x_{w m}^{*}<x_{l m}^{*}$, the 3 feasible orderings correspond to either $x_{w m}^{*}$ being less than both $x_{w c}^{*}$ and $x_{w p}^{*}$, lying between $x_{w c}^{*}$ and $x_{w p}^{*}$, or greater than both $x_{w c}^{*}$ and $x_{w p}^{*}$.

(a) The first case is

$$
x_{w m}^{*}<x_{l m}^{*}<x_{w c}^{*}<x_{w p}^{*},
$$


so consequently $x_{w s}^{*}=x_{l m}^{*}$. In this case, $x_{w s}^{*}<x_{w c}^{*}$. The new product is not commercialized immediately so sleeping patent occurs.

(b) The second case corresponds to

$$
x_{w m}^{*}<x_{w c}^{*}<x_{l m}^{*}<x_{w p}^{*},
$$

and consequently $x_{w s}^{*}=x_{l m}^{*}$. The winner firm epsilon preempts its rival. The new substitute product is commercialized immediately since $x_{w s}^{*}>x_{w c}^{*}$, so sleeping patent does not occur.

(c) The last case is given by

$$
x_{w m}^{*}<x_{w c}^{*}<x_{w p}^{*}<x_{l m}^{*},
$$

where "sleeping patent" does not occur and $x_{w s}^{*}=x_{w p}^{*}$. The winner firm exercises the option of patenting at its optimal patenting threshold without any preemptive threat from its rival.

The above results are summarized in Table 1.

In the next section, we consider the conditions on the relative magnitude of commercialization costs and the profit flow rates of the two firms that determine (i) which firm is the winner in the patent race, (ii) whether the winner chooses to let the patent sleep.

\section{Characterization of the preemption strate- gies and sleeping patent}

In Section 3, we show that sleeping patent occurs if and only if the winner's commercialization threshold $x_{w c}^{*}$ is higher than or equal to the loser's Marshallian trigger $x_{l m}^{*}$. Next, we would like to investigate how the cost and profit flow parameters determine the winner of the game. Before we determine which firm is the winner, it would be instructive to establish the conditions on the costs and profit flow rates such that (i) $x_{i m}^{*} \leq x_{e c}^{*}$, (ii) $x_{e m}^{*} \leq x_{i c}^{*}$. The results are summarized in Proposition 1.

Proposition 1 Let $z$ be the (unique) solution to the equation: 


$$
f(z)=\frac{K_{i}}{\beta-1}\left(\frac{\pi_{i}^{+}}{K_{i} \pi_{e}}\right)^{\beta} z^{\beta}+\frac{\beta}{\beta-1} \frac{\pi_{i}^{-}}{\pi_{e}} z-C=0 .
$$

Also, we define

$$
K_{i}^{*}=\frac{C(\beta-1) \pi_{i}^{+}}{\pi_{i}^{+}+\beta \pi_{i}^{-}} \text {and } K_{i}^{* *}=\frac{C(\beta-1) \pi_{i}^{+}}{\pi_{e}}
$$

(a) The conditions on $K_{i}$ and $K_{e}$ such that $x_{i m}^{*} \leq x_{e c}^{*}$ are given by

$$
x_{i m}^{*} \leq x_{e c}^{*} \Leftrightarrow\left\{\begin{array}{ll}
K_{e} \geq \frac{\beta-1}{\beta} \frac{\pi_{e}\left(K_{i}+C\right)}{\pi_{i}^{+}+\pi_{i}^{-}} & \text {for } K_{i} \leq K_{i}^{*} \\
K_{e} \geq z & \text { for } K_{i}>K_{i}^{*}
\end{array} .\right.
$$

(b) The conditions on $K_{i}$ and $K_{e}$ such that $x_{e m}^{*} \leq x_{i c}^{*}$ are given by

$$
x_{e m}^{*} \leq x_{i c}^{*} \Leftrightarrow\left\{\begin{array}{ll}
K_{e} \leq \frac{\beta}{\beta-1} \frac{\pi_{e} K_{i}}{\pi_{i}^{+}}-C & \text { for } K_{i} \leq K_{i}^{* *} \\
K_{e} \leq\left[\left(\frac{\pi_{e} K_{i}}{\pi_{i}^{+}}\right)^{\beta} \frac{1}{C(\beta-1)}\right]^{\frac{1}{\beta-1}} & \text { for } K_{i}>K_{i}^{* *}
\end{array} .\right.
$$

The proof of Proposition 1 is presented in Appendix A.

In Proposition 2, we state the conditions on the costs and profit flow rates such that (i) $x_{i m}^{*} \leq x_{e p}^{*}$, (ii) $x_{e m}^{*} \leq x_{i p}^{*}$. The results provide the preliminary procedure that determines whether the winner chooses to exercise the option of acquiring the patent by $\varepsilon$-preemption or at the optimal non-competitive patent trigger.

\section{Proposition 2}

(a) The conditions on $K_{i}$ and $K_{e}$ such that $x_{i m}^{*} \leq x_{e p}^{*}$ are given by

$$
x_{i m}^{*} \leq x_{e p}^{*} \Leftrightarrow\left\{\begin{array}{ll}
K_{e} \geq \frac{\beta-1}{\beta}\left[\frac{\pi_{e}\left(K_{i}+C\right)}{\pi_{i}^{+}+\pi_{i}^{-}}\right]-C & \text { for } K_{i} \leq K_{i}^{*} \\
K_{e} \geq z-C & \text { for } K_{i}>K_{i}^{*}
\end{array} .\right.
$$

(b) The conditions on $K_{i}$ and $K_{e}$ such that $x_{e m}^{*} \leq x_{i p}^{*}$ are given by

$$
x_{e m}^{*} \leq x_{i p}^{*} \Leftrightarrow \begin{cases}K_{e} \leq \frac{\beta}{\beta-1} \frac{\pi_{e}\left(K_{i}+C\right)}{\pi_{i}^{+}}-C & \text { for } K_{i} \leq K_{i}^{* *}-C \\ K_{e} \leq\left\{\left[\frac{\pi_{e}\left(K_{i}+C\right)}{\pi_{i}^{+}}\right]^{\beta} \frac{1}{C(\beta-1)}\right\}^{\frac{1}{\beta-1}} & \text { for } K_{i}>K_{i}^{* *}-C\end{cases}
$$


The derivation procedure required to establish Proposition 2 can be mimicked from that in the proof of Proposition 1.

Most importantly, we need to determine which firm is the winner in this patent race, where the winner is the firm with the lower Marshallian trigger. The relative ordering of the two Marshallian triggers depends on the commercialization costs and profit flow rates of the two competing firms. Apparently, the firm with the lower sunk cost and higher profit rate would be likely to be the winner in this game. Now, we present the conditions on $K_{i}$ and $K_{e}$ that determine the winner. These conditions depend on the magnitude of $\pi_{e}$ relative to $\beta \pi_{i}^{-}$and $\beta \pi_{i}^{-}+\pi_{i}^{+}$. The relevant results are summarized in Proposition 3.

Proposition 3 In the parameter space of $K_{i}$ and $K_{e}$, the boundary curves that determine the ordering of $x_{e m}^{*}$ and $x_{i m}^{*}$ are characterized by the following conditions on the cost and profit flow rate parameters.

(a) When $\pi_{e}>\beta \pi_{i}^{-}+\pi_{i}^{+}$, we have $x_{e m}^{*} \leq x_{i m}^{*}$

$\Leftrightarrow\left\{\begin{array}{ll}K_{e} \leq \frac{\pi_{e} K_{i}}{\pi_{i}^{+}}\left(\frac{\pi_{e}-\beta \pi_{i}^{-}}{\pi_{i}^{+}}\right)^{\frac{1}{\beta-1}} & \text { for } K_{i} \geq C(\beta-1)\left(1-\frac{\beta \pi_{i}^{-}}{\pi_{e}}\right) \\ K_{e} \leq\left[\left(\frac{\pi_{e}}{\pi_{i}^{+}}\right)^{\beta} \frac{(\beta-1)^{\beta-1}}{\beta^{\beta} C}\left(K_{i}+C-\frac{\beta C \pi_{i}^{-}}{\pi_{e}}\right)^{\beta}\right]^{\frac{1}{\beta-1}} & \text { for } C(\beta-1)\left(1-\frac{\beta \pi_{i}^{-}}{\pi_{e}}\right)>K_{i} \geq C\left(\frac{\beta\left(\pi_{i}^{+}+\pi_{i}^{-}\right)}{\pi_{e}}-1\right) \\ K_{e} \leq \frac{\pi_{e}\left(K_{i}+C\right)}{\pi_{i}^{+}+\pi_{i}^{-}}-C & \text { for } K_{i}<C\left(\frac{\beta\left(\pi_{i}^{+}+\pi_{i}^{-}\right)}{\pi_{e}}-1\right)\end{array}\right.$.

(b) When $\beta \pi_{i}^{-}+\pi_{i}^{+} \geq \pi_{e}>\beta \pi_{i}^{-}$, we have $x_{e m}^{*} \leq x_{i m}^{*}$

$\Leftrightarrow \begin{cases}K_{e} \leq \frac{\pi_{e} K_{i}}{\pi_{i}^{+}}\left(\frac{\pi_{e}-\beta \pi_{i}^{-}}{\pi_{i}^{+}}\right)^{\frac{1}{\beta-1}} & \text { for } K_{i} \geq\left[\frac{C(\beta-1) \pi_{i}^{+}}{\pi_{e}}\right]^{\frac{1}{\beta-1}} \frac{\pi_{i}^{+}}{\pi_{e}-\beta \pi_{i}^{-}} \\ K_{e} \leq \hat{z}-C & \text { for }\left[\frac{C(\beta-1) \pi_{i}^{+}}{\pi_{e}}\right]^{\frac{1}{\beta-1}} \frac{\pi_{i}^{+}}{\pi_{e}-\beta \pi_{i}^{-}}>K_{i} \geq \frac{C(\beta-1) \pi_{i}^{+}}{\beta \pi_{i}^{-}+\pi_{i}^{+}} \\ K_{e} \leq \frac{\pi_{e}\left(K_{i}+C\right)}{\pi_{i}^{+}+\pi_{i}^{-}}-C & \text { for } K_{i}<\frac{C(\beta-1) \pi_{i}^{+}}{\beta \pi_{i}^{-}+\pi_{i}^{+}}\end{cases}$

where $\hat{z}$ is the (unique) solution to the equation

$$
g(z)=\frac{K_{i}}{\beta-1}\left(\frac{\beta-1}{\beta} \frac{\pi_{i}^{+}}{K_{i} \pi_{e}}\right)^{\beta} z^{\beta}+\frac{\pi_{i}^{-}}{\pi_{e}} z-C=0 .
$$


(c) When $\pi_{e} \leq \beta \pi_{i}^{-}$, we have

$x_{e m}^{*} \leq x_{i m}^{*}$

$\Leftrightarrow\left\{\begin{array}{ll}K_{e} \leq \hat{z}-C & \text { for } K_{i} \geq \frac{C(\beta-1) \pi_{i}^{+}}{\beta \pi_{i}^{-}+\pi_{i}^{+}} \\ K_{e} \leq \frac{\pi_{e}\left(K_{i}+C\right)}{\pi_{i}^{+}+\pi_{i}^{-}}-C & \text { for } K_{i}<\frac{C(\beta-1) \pi_{i}^{+}}{\beta \pi_{i}^{-}+\pi_{i}^{+}}\end{array}\right.$.

The proof of Proposition 3 is presented in Appendix B.

Once we have established the conditions on the commercialization costs and profit flow rates that determine the relative ordering of the patent triggers, commercialization triggers and Marshallian triggers of the two competing firms, we then examine the characterization of the strategies on preemptive patenting adopted by the incumbent and entrant and the sleeping patent phenomena in the two-dimensional $K_{i}-K_{e}$ space. In our sample calculations for generating the plots that demonstrate the behavior of preemptive patenting and sleeping patent, the following set of parameter values are adopted: $r=0.1, \mu=0.01, C=5, \sigma=0.3, \pi_{i}^{+}=1, \pi_{i}^{-}=0.1, \pi_{e}=1.5$.

In Figure 1, we illustrate the characterization of the preemptive patenting behavior of the two competing firms with regard to the relative magnitude of their commercialization costs. We plot the boundary curves in the $K_{i^{-}}$ $K_{e}$ plane that separate the various regions where the incumbent wins or the entrant wins. To the top left region above the middle thick curve where $x_{i m}^{*}=x_{e m}^{*}$, the more cost efficient incumbent is the winner of the patent race since $x_{i m}^{*}<x_{e m}^{*}$. The incumbent wins by either $\varepsilon$-preempting the entrant or exercising the patent option optimally without preemptive threat from its less efficient rival. The $\varepsilon$-preemption case corresponds to the scenario where $x_{i m}^{*}<x_{e m}^{*}<x_{i p}^{*}$, which is represented by the region that is bounded between the boundary curves: $x_{i m}^{*}=x_{e m}^{*}$ and $x_{i p}^{*}=x_{e m}^{*}$. Since the incumbent wins by $\varepsilon$-preemption, so $x_{i s}^{*}=x_{e m}^{*}-\varepsilon$. The "no preemptive threat" case corresponds to the scenario where $x_{i m}^{*}<x_{i p}^{*}<x_{e m}^{*}$, which is represented by the region that lies to the top left side above the boundary curve: $x_{i p}^{*}=x_{e m}^{*}$. In this case, the entrant is highly cost inefficient and the incumbent chooses to acquire the patent optimally at $x_{i p}^{*}$. On the other hand, in the region to the lower right side below the middle thick curve $x_{i m}^{*}=x_{e m}^{*}$, the entrant is more cost efficient. In a similar manner, we deduce that the strategic preemption trigger $x_{e s}^{*}$ of the entrant, which is now the winner firm, can be either $x_{e s}^{*}=x_{i m}^{*}-\varepsilon$ or $x_{e s}^{*}=x_{e p}^{*}$, corresponding to the region where $x_{e m}^{*}<x_{i m}^{*}<x_{e p}^{*}$ or $x_{e m}^{*}<x_{e p}^{*}<x_{i m}^{*}$, respectively. 
It may be instructive to examine the dependence of the strategic preemption triggers of the two firms on the commercialization costs. In Figure 2, we show the plot of $x_{e s}^{*}$ against $K_{e}$, assuming $K_{i}=10$. When $K_{e}$ is small such that $K_{e}<z-c$, the entrant is highly cost efficient (see Proposition 2, part a). The entrant wins by exercising the patent option without preemptive threat from its rival and so $x_{e s}^{*}=x_{e p}^{*}$. When $K_{e}$ increases beyond the value $z-c$ but $K_{e}<\frac{\pi_{e} K_{i}}{\pi_{i}^{+}}\left(\frac{\pi_{e}-\beta \pi_{i}^{-}}{\pi_{i}^{+}}\right)^{\frac{1}{\beta-1}}$, the entrant faces the preemption threat from the incumbent. In this case, the entrant wins by $\varepsilon$-preemption so that $x_{e s}^{*}=x_{i m}^{*}-\varepsilon$. Actually, at the critical value where $K_{e}=\frac{\pi_{e} K_{i}}{\pi_{i}^{+}}\left(\frac{\pi_{e}-\beta \pi_{i}^{-}}{\pi_{i}^{+}}\right)^{\frac{1}{\beta-1}}$, the Marshallian trigger of the two firms are equal (see Proposition 3, part a) and each firm has $50 \%$ chance to win. When $K_{e}$ increases further and stays above the critical value, the entrant becomes the loser firm and $x_{e s}^{*}$ is not defined. In summary, we have

$$
x_{e s}^{*}= \begin{cases}x_{e p}^{*} & \text { for } 0<K_{e}<z-C \\ x_{i m}^{*}-\varepsilon & \text { for } z-C \leq K_{e}<\frac{\pi_{e} K_{i}}{\pi_{i}^{+}}\left(\frac{\pi_{e}-\beta \pi_{i}^{-}}{\pi_{i}^{+}}\right)^{\frac{1}{\beta-1}} .\end{cases}
$$

Similarly, we show the plot of $x_{i s}^{*}$ against $K_{e}$ in Figure 3 , assuming $K_{i}=$ 10. The incumbent firm wins the patent race only if $K_{e}>\frac{\pi_{e} K_{i}}{\pi_{i}^{+}}\left(\frac{\pi_{e}-\beta \pi_{i}^{-}}{\pi_{i}^{+}}\right)^{\frac{1}{\beta-1}}$. The incumbent firm $\varepsilon$-preempts its rival so that $x_{i s}^{*}=x_{e m}^{*}-\varepsilon$ when

$$
\frac{\pi_{e} K_{i}}{\pi_{i}^{+}}\left(\frac{\pi_{e}-\beta \pi_{i}^{-}}{\pi_{i}^{+}}\right)^{\frac{1}{\beta-1}}<K_{e} \leq\left\{\left[\frac{\pi_{e}\left(K_{i}+C\right)}{\pi_{i}^{+}}\right]^{\beta} \frac{1}{C(\beta-1)}\right\}^{\frac{1}{\beta-1}}
$$

and exercises the patent option optimally at $x_{i p}^{*}$ when

$$
K_{e}>\left\{\left[\frac{\pi_{e}\left(K_{i}+C\right)}{\pi_{i}^{+}}\right]^{\beta} \frac{1}{C(\beta-1)}\right\}^{\frac{1}{\beta-1}}
$$

(see Proposition 2, part b). In summary, we have

$$
x_{i s}^{*}= \begin{cases}x_{e m}^{*}-\varepsilon & \text { for } \frac{\pi_{e} K_{i}}{\pi_{i}^{+}}\left(\frac{\pi_{e}-\beta \pi_{i}^{-}}{\pi_{i}^{+}}\right)^{\frac{1}{\beta-1}}<K_{e} \leq\left\{\left[\frac{\pi_{e}\left(K_{i}+C\right)}{\pi_{i}^{+}}\right]^{\beta} \frac{1}{C(\beta-1)}\right\}^{\frac{1}{\beta-1}} \\ x_{i p}^{*} & \text { for } K_{e}>\left\{\left[\frac{\pi_{e}\left(K_{i}+C\right)}{\pi_{i}^{+}}\right]^{\beta} \frac{1}{C(\beta-1)}\right\}^{\frac{1}{\beta-1}}\end{cases}
$$


Finally, we also plot $x_{e s}^{*}\left(\right.$ with $\left.K_{e}=10\right)$ and $x_{i s}^{*}$ (with $\left.K_{e}=30\right)$ against $K_{i}$ in Figure 4 and Figure 5, respectively. The plots confirm with the following results that can be deduced from Propositions 2 and 3:

$$
x_{e s}^{*}=\left\{\begin{array}{ll}
x_{i m}^{*}-\varepsilon & \text { for } \frac{\pi_{i}^{+} K_{e}}{\pi_{e}}\left(\frac{\pi_{i}^{+}}{\pi_{e}-\beta \pi_{i}^{-}}\right)^{\frac{1}{\beta-1}}<K_{i} \leq \frac{\beta\left(K_{e}+C\right)}{\beta-1} \frac{\pi_{i}^{+}+\pi_{i}^{-}}{\pi_{e}}-C \\
x_{e p}^{*} & \text { for } K_{i}>\frac{\beta\left(K_{e}+C\right)}{\beta-1} \frac{\pi_{i}^{+}+\pi_{i}^{-}}{\pi_{e}}-C
\end{array} ;\right.
$$

and

$x_{i s}^{*}= \begin{cases}x_{i p}^{*} & \text { for } 0<K_{i}<\frac{\pi_{i}^{+}}{\pi_{e}}\left[C(\beta-1) K_{e}^{\beta-1}\right]^{\frac{1}{\beta}}-C \\ x_{e m}^{*}-\varepsilon & \text { for } \frac{\pi_{i}^{+}}{\pi_{e}}\left[C(\beta-1) K_{e}^{\beta-1}\right]^{\frac{1}{\beta}}-C \leq K_{i}<\frac{\pi_{i}^{+} K_{e}}{\pi_{e}}\left(\frac{\pi_{i}^{+}}{\pi_{e}-\beta \pi_{i}^{-}}\right)^{\frac{1}{\beta-1}} .\end{cases}$

It is now relatively straightforward to deduce the regions in the $K_{i^{-}}$ $K_{e}$ plane as shown in Figure 6 such that sleeping patent occurs. When $x_{i m}^{*}<x_{e m}^{*}$, the incumbent is the winner; the incumbent lets the patent sleep when $x_{e m}^{*} \leq x_{i c}^{*}$ [see Eq.(3.12)]. Hence, the region in the $K_{i}-K_{e}$ plane when the incumbent lets the patent sleep is bounded between the boundary curves: $x_{e m}^{*}=x_{i c}^{*}$ and $x_{e m}^{*}=x_{i m}^{*}$ (see the upper shaded region in Figure 6). Similarly, the entrant lets the patent sleep when $x_{e m}^{*}<x_{i m}^{*} \leq x_{e c}^{*}$, corresponding to the lower shaded region in Figure 6 . We then obtain the full characterization of the sleeping patent phenomenon in the parameter space of the cost parameters $K_{i}$ and $K_{e}$ of the two competing firms.

\section{Conclusion}

We have applied a real options game model to identify the incentive for preemptive patenting between two asymmetric firms. There are three forms of asymmetry in our patent-investment race model. The status of one firm is incumbent while the other is a new entrant, the commercialization costs of the substitute product are different, and the profit flow rates resulting from launching of the substitute product are not the same for the two firms. The incumbent receives the monopolized profit flow from operating an existing product in the market, but the monopoly may be undermined when the challenger firm (new entrant) obtains the patent and later commercializes the product. Either firm may choose to preempt its competitor provided that the patent cost is less than the benefit gained by preventing competitor's entry. 
In our two-stage patent-investment race model, we show that the strategic preemption trigger always lies between the Marshallian trigger and the firm's optimal non-strategic patent trigger. The firm with the lower Marshallian trigger is considered to be the efficient firm. The fear of preemption is seen to undermine part of the real option value of investment. Under certain conditions, the efficient firm may choose to "epsilon preempt" the competitor while still preserves some real option value of waiting. The act of preemptive patenting by the efficient firm occurs when the Marshallian trigger of the less efficient firm is lower than the non-strategic patent trigger of the efficient firm.

The relative magnitude of the costs and profit flow rates among the two firms in our two-stage patent-investment race model determines which firm is the winner of the patent race. We manage to provide the full characterization of the optimal strategies taken by the competing firms in terms of the cost and profit flow rate parameters.

Our patent-investment game model provides an analytic framework to explore the scenarios under which sleeping patent may occur. The patent is acquired at a lower threshold of preemptive patenting and it is kept unexploited for a period of time. This occurs when the preemptive patent trigger of the efficient firm is lower than its optimal non-strategic commercialization trigger. The winner firm would rush to acquire the patent at a lower strategic preemption trigger (thus letting the patent sleep for a longer duration) when it faces higher preemptive threat from its competitor (as revealed by a lower Marshallian trigger of the loser firm).

\section{Acknowledgment}

The authors would like to thank Miss Ka Man Chan for her contributions to the preliminary analysis of the patent race model.

\section{References}

[1] Dixit, A., Pindyck, R., 1994. Investment under uncertainty. Princeton University Press, Princeton, New Jersey.

[2] Gilbert, R.J., Newbery, D.M.G., 1982. Preemptive patenting and the persistence of monopoly, American Economic Review, 72(3), 514-526. 
[3] Grenadier, S.R., 1996. The strategic exercise of options: Development cascades and overbuilding in real estate markets, Journal of Finance, 51, 1653-1679.

[4] Harris, C., Vickers, J., 1985. Patent races and the persistence of monopoly, Journal of Industrial Economics, 33, 461-481.

[5] Hsu, Y.W., Lambrecht, B.M., 2007. Preemptive patenting under uncertainty and asymmetric information, Annals of Operations Research, 151(1), 5-28.

[6] Kong, J.J., Kwok, Y.K., 2007. Real options in strategic investment games betweeen two asymmetric firms, European Journal of Operational Research, 181, p.967-985.

[7] Lambrecht, B., 2000. Strategic sequential investments and sleeping patents, in Project Flexibility, Agent and Competition: New Developments in the Theory and Application of Real Options, edited by Brennan, M.J. and Trigeorgis, L., Oxford University Press, Oxford.

[8] Pakes, A., 1986. Patents as options: Some estimates of the value of holding European patent shocks, Econometrica, 54(4), 755-784.

[9] Palomeras, N., 2003. Sleeping patents: Any reason to wake up, Working paper of the University of Navarra.

[10] Reiss, A., 1998. Investment in innovations and competition: An option pricing approach, Quarterly Review of Economics and Finance, 38, 635650 .

[11] Sereno, L., 2008. Real options and economic valuation of patents, Working paper of University of Bologna.

[12] Takalo, T., Kanniainen, V., 2000. Do patents slow down technological progress: Real options in research, patenting and market introduction, International Journal of Industrial Organization, 18, 1105-1127.

[13] Weeds, H., 1999. Sleeping patents and compulsory licensing: An options analysis, Working paper of the University of Warwick. 


\section{Appendix A - Proof of Proposition 1}

Since $\Phi_{i}\left(x_{i m}^{*}\right)=0$ and $\Phi_{i}(x)$ is strictly increasing in $x$, we have

$$
x_{i m}^{*} \leq x_{e c}^{*} \Leftrightarrow \Phi_{i}\left(x_{e c}^{*}\right) \geq 0,
$$

where

$$
\Phi_{i}\left(x_{e c}^{*}\right)=\left\{\begin{array}{ll}
\frac{\pi_{i}^{+} x_{e c}^{*}}{r-\mu}-K_{i}-C+\frac{\pi_{i}^{-} x_{e c}^{*}}{r-\mu} \geq 0 & \text { for } x_{i c}^{*} \leq x_{e c}^{*} \\
\left(\frac{\pi_{i}^{+} x_{i c}^{*}}{r-\mu}-K_{i}\right)\left(\frac{x_{e c}^{*}}{x_{i c}^{*}}\right)^{\beta}-C+\frac{\pi_{i}^{-} x_{e c}^{*}}{r-\mu} \geq 0 & \text { for } x_{i c}^{*}>x_{e c}^{*}
\end{array} .\right.
$$

On the other hand, the relative ordering of $x_{i c}^{*}$ and $x_{e c}^{*}$ depends on the relative magnitude of the commercialization costs where

$$
x_{i c}^{*} \leq x_{e c}^{*} \Leftrightarrow K_{i} \leq \frac{\pi_{i}^{+} K_{e}}{\pi_{e}} .
$$

Substituting Eq. (A.2) into Eq. (A.1), we obtain

$$
x_{i m}^{*} \leq x_{e c}^{*} \Leftrightarrow\left\{\begin{array}{ll}
K_{e} \geq \frac{\beta-1}{\beta} \frac{\pi_{e}\left(K_{i}+C\right)}{\pi_{i}^{+}+\pi_{i}^{-}} & \text {for } K_{e} \geq \frac{\pi_{e} K_{i}}{\pi_{i}^{+}} \\
K_{e} \geq z & \text { for } K_{e}<\frac{\pi_{e} K_{i}}{\pi_{i}^{+}}
\end{array},\right.
$$

where $z$ is the unique solution to the equation $f(z)=0$. Note that $z$ has dependence on $K_{i}$. The above pair of inequalities define the region in the $K_{i}-K_{e}$ plane satisfying $x_{i m}^{*} \leq x_{e c}^{*}$. After some algebraic manipulation, one can show that

$$
x_{i m}^{*} \leq x_{e c}^{*} \Leftrightarrow\left\{\begin{array}{ll}
K_{e} \geq \frac{\beta-1}{\beta} \frac{\pi_{e}\left(K_{i}+C\right)}{\pi_{i}^{+}+\pi_{i}^{-}} & \text {for } K_{i} \leq \frac{C(\beta-1) \pi_{i}^{+}}{\pi_{i}^{+}+\beta \pi_{i}^{-}} \\
K_{e} \geq z & \text { for } K_{i}>\frac{C(\beta-1) \pi_{i}^{+}}{\pi_{i}^{+}+\beta \pi_{i}^{-}}
\end{array} .\right.
$$

The proof for establishing the conditions on $K_{i}$ and $K_{e}$ such that $x_{e m}^{*} \leq x_{i c}^{*}$ can be performed in a similar manner.

\section{Appendix B - Proof of Proposition 3}

Similar to the proof of Proposition 1, we first deduce that

$$
x_{e m}^{*} \leq x_{i m}^{*} \Leftrightarrow V_{i}^{(2)}\left(x_{e m}^{*}\right)-C-L_{i}\left(x_{e m}^{*}\right) \leq 0 .
$$


Recall that the value function $V_{i}^{(2)}(x)$ has two analytic functional forms with regard to the relative magnitude of $x_{e m}^{*}$ and $x_{i c}^{*}$. Also, the various conditions on the cost and profit flow rate parameters that determine the order of $x_{e m}^{*}$ and $x_{i c}^{*}$ have been established in Proposition 1. Similarly, the loss function $L_{i}(x)$ has two analytic functional forms with regard to the relative magnitude of $x_{e m}^{*}$ and $x_{e c}^{*}$, and

$$
x_{e m}^{*} \geq x_{e c}^{*} \Leftrightarrow K_{e} \leq C(\beta-1) .
$$

We then have

$$
\Leftrightarrow\left\{\begin{array}{l}
x_{e m}^{*} \leq x_{i m}^{*} \\
\qquad \begin{array}{l}
\begin{array}{l}
\frac{\pi_{i}^{+} x_{e m}^{*}}{r-\mu}-K_{i}-C+\frac{\pi_{i}^{-} x_{e m}^{*}}{r-\mu} \leq 0 \\
\left(\frac{\pi_{i}^{+} x_{i c}^{*}}{r-\mu}-K_{i}\right)\left(\frac{x_{e m}^{*}}{x_{i c}^{*}}\right)^{\beta}-C+\frac{\pi_{i}^{-} x_{e m}^{*}}{r-\mu} \leq 0 \\
\text { if } K_{e} \leq C(\beta-1) \text { and } x_{e m}^{*} \geq x_{i c}^{*} \\
\frac{\pi_{i}^{+} x_{e m}^{*}}{r-\mu}-K_{i}-C+\frac{\pi_{i}^{-} x_{e c}^{*}}{r-\mu}\left(\frac{x_{e m}^{*}}{x_{e c}^{*}}\right)^{\beta_{0}} \leq 0 \\
\text { if } K_{e}>C(\beta-1) \text { and } x_{e m}^{*}<x_{i c}^{*} \\
\left(\frac{\pi_{i}^{+} x_{i c}^{*}}{r-\mu}-K_{i}\right)\left(\frac{x_{e m}^{*}}{x_{i c}^{*}}\right)^{\beta}-C+\frac{\pi_{i}^{-} x_{e c}^{*}}{r-\mu}\left(\frac{x_{e m}^{*}}{x_{e c}^{*}}\right)^{\beta} \leq 0 \\
\text { if } K_{e}>C(\beta-1) \text { and } x_{e m}^{*} \geq x_{i c}^{*}
\end{array} . x_{i c}^{*}
\end{array}
\end{array}\right.
$$

It is necessary to consider the various regions in the parameter space of $K_{i}$ and $K_{e}$ when we derive the conditions on the cost and profit flow rate parameters such that $x_{e m}^{*} \leq x_{i m}^{*}$.

As an illustrative example, we consider the set of conditions on the cost parameters under which $x_{e m}^{*} \leq x_{i m}^{*}$ when $K_{i}>\frac{C(\beta-1) \pi_{i}^{+}}{\pi_{e}}$. Under this assumption, we recall from Proposition 1 that

$$
x_{e m}^{*} \leq x_{i c}^{*} \Leftrightarrow K_{e} \leq\left[\left(\frac{\pi_{e} K_{i}}{\pi_{i}^{+}}\right)^{\beta} \frac{1}{C(\beta-1)}\right]^{\frac{1}{\beta-1}} .
$$

As part of the derivation procedure, it is important to observe

$$
\left[\left(\frac{\pi_{e} K_{i}}{\pi_{i}^{+}}\right)^{\beta} \frac{1}{C(\beta-1)}\right]^{\frac{1}{\beta-1}}>C(\beta-1) .
$$

After some tedious manipulation with the algebraic inequalities, we obtain (i) For $0 \leq K_{e} \leq C(\beta-1)$ 


$$
x_{e m}^{*} \leq x_{i m}^{*} \Leftrightarrow \begin{cases}K_{e} \leq \hat{z}-C & \text { if } g(C \beta) \geq 0 \\ K_{e} \leq C(\beta-1) & \text { if } g(C \beta)<0\end{cases}
$$

where $\hat{z}$ is the unique solution to $g(z)=0$.

(ii) For $C(\beta-1)<K_{e} \leq\left[\left(\frac{\pi_{e} K_{i}}{\pi_{i}^{+}}\right)^{\beta} \frac{1}{C(\beta-1)}\right]^{\frac{1}{\beta-1}}$,

$$
x_{e m}^{*} \leq x_{i m}^{*} \Leftrightarrow\left\{\begin{array}{c}
C(\beta-1) \leq K_{e} \leq \frac{\pi_{e} K_{i}}{\pi_{i}^{+}}\left(\frac{\pi_{e}-\beta \pi_{i}^{-}}{\pi_{i}^{+}}\right){ }^{\frac{1}{\beta-1}} \\
\text { if } g(C \beta) \leq 0 \text { and } K_{i} \geq C(\beta-1)\left(1-\frac{\beta \pi_{i}^{-}}{\pi_{e}}\right) \\
C(\beta-1) \leq K_{e} \leq\left[\left(\frac{\pi_{e} K_{i}}{\pi_{i}^{+}}\right)^{\beta} \frac{1}{C\left(\beta_{0}-1\right)}\right]^{\frac{1}{\beta-1}} \\
\text { if } g(C \beta) \leq 0 \text { and } K_{i} \leq C(\beta-1)\left(1-\frac{\beta \pi_{i}^{-}}{\pi_{e}}\right)
\end{array} .\right.
$$

(iii) For $K_{e}>\left[\left(\frac{\pi_{e} K_{i}}{\pi_{i}^{+}}\right)^{\beta} \frac{1}{C(\beta-1)}\right]^{\frac{1}{\beta-1}}$

$$
\begin{aligned}
& x_{e m}^{*} \leq x_{i m}^{*} \Leftrightarrow \quad\left[\left(\frac{\pi_{e} K_{i}}{\pi_{i}^{+}}\right)^{\beta} \frac{1}{C(\beta-1)}\right]^{\frac{1}{\beta-1}} \leq K_{e}<D \\
& \text { if } K_{i}<C\left(\beta_{0}-1\right)\left(1-\frac{\beta \pi_{i}^{-}}{\pi_{e}}\right)
\end{aligned}
$$

where $D=\left[\left(\frac{\pi_{e}}{\pi_{i}^{+}}\right)^{\beta} \frac{(\beta-1)^{\beta-1}}{\beta^{\beta} C}\left(K_{i}+C-\frac{\beta \pi_{i}^{-} C}{\pi_{e}}\right)^{\beta}\right]^{\frac{1}{\beta-1}}$.

Similarly, when $K_{i} \leq \frac{C(\beta-1) \pi_{i}^{+}}{\pi_{e}}$, we can also deduce another set of conditions on the cost parameters under which $x_{e m}^{*} \leq x_{i m}^{*}$. Furthermore, we need to establish the following conditions under which $g(C \beta) \geq 0$ :

(i) If $\pi_{e} \leq \beta \pi_{i}^{-}$, then $g(C \beta) \geq 0$.

(ii) If $\pi_{e}>\beta \pi_{i}^{-}$, then we have

$$
g(C \beta) \geq 0 \Leftrightarrow K_{i} \leq \frac{C(\beta-1) \pi_{i}^{+}}{\pi_{e}}\left(\frac{\pi_{i}^{+}}{\pi_{e}-\beta \pi_{i}^{-}}\right)^{\frac{1}{\beta-1}} .
$$

Finally, we combine all of the above conditions on the algebraic inequalities under which $x_{e m}^{*} \leq x_{i m}^{*}$ and obtain the results as stated in Proposition 3. 


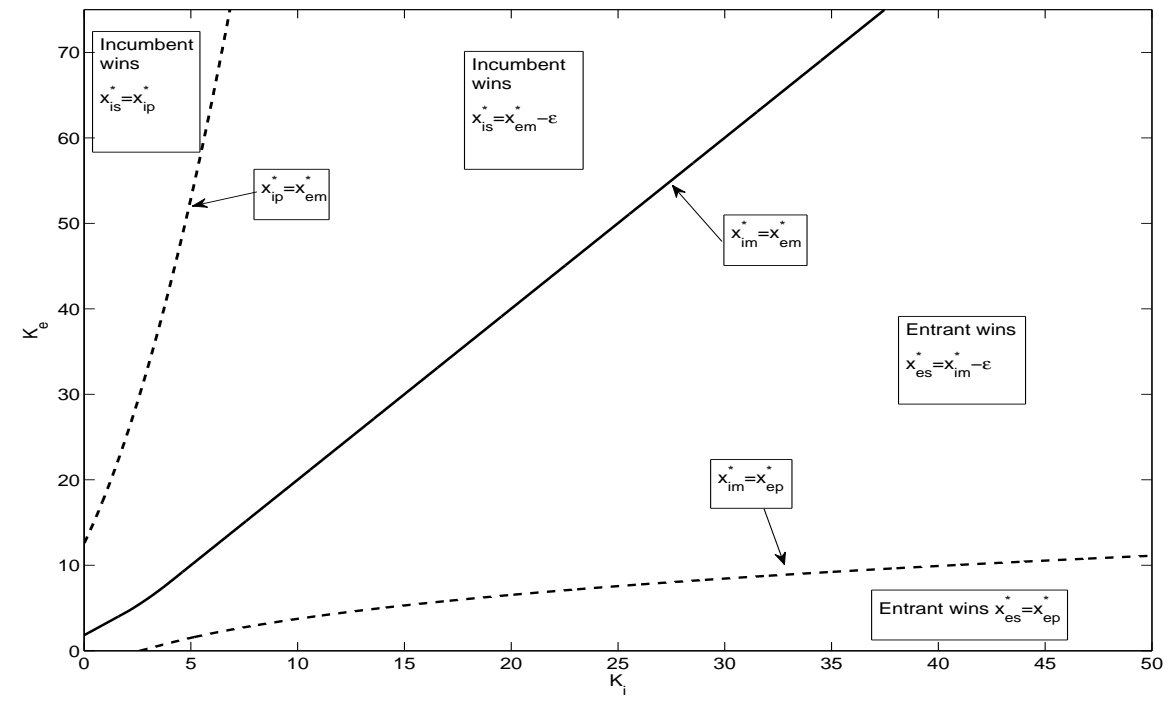

Figure 1: Characterization of the preemptive patenting behavior of the two competing firms in the $K_{i}-K_{e}$ plane.

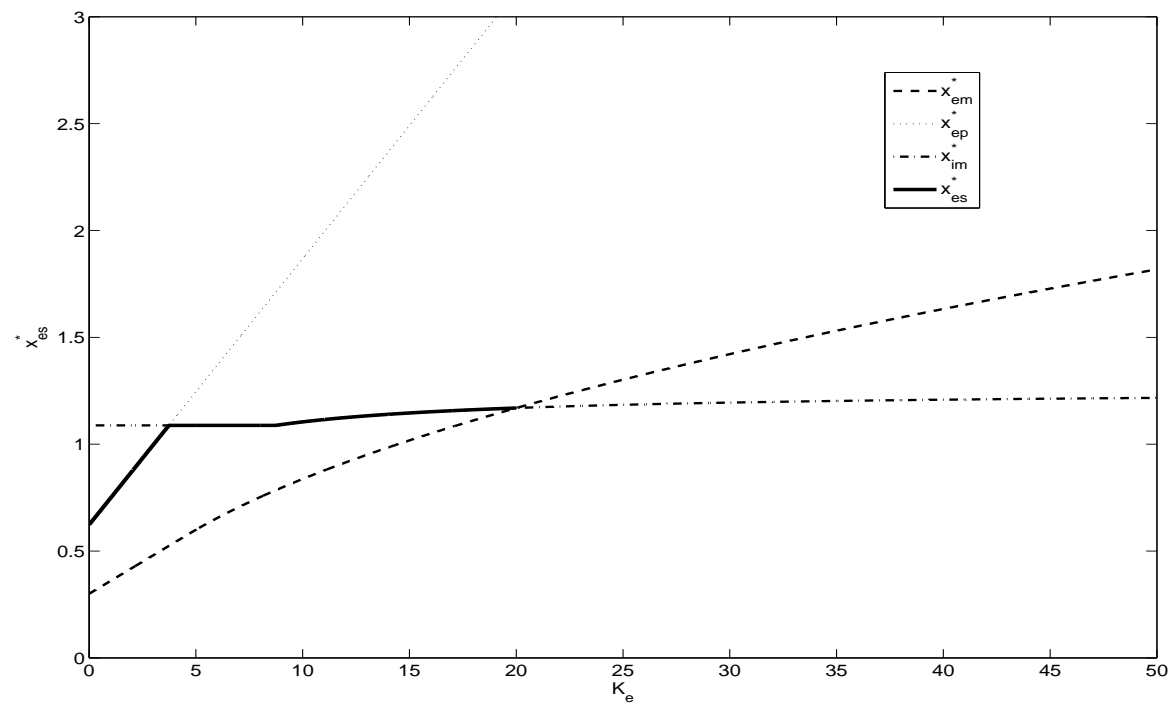

Figure 2: Plot of the entrant's strategic preemption trigger $x_{e s}^{*}$ against $K_{e}$. 


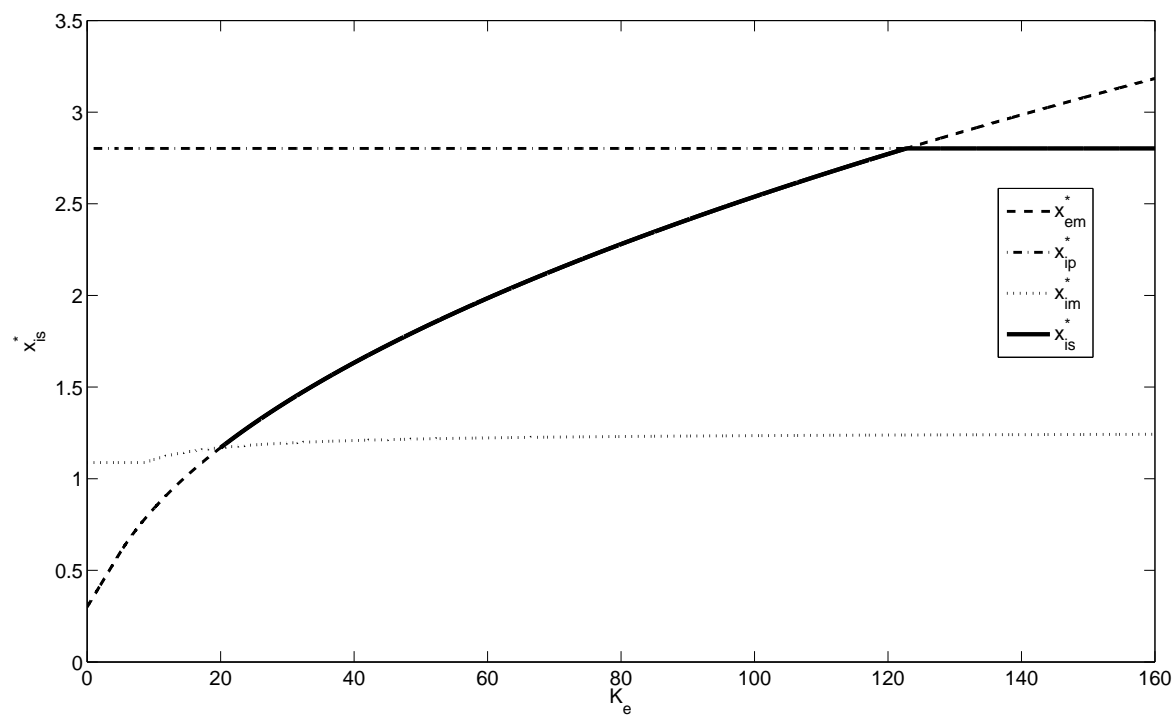

Figure 3: Plot of the incumbent's strategic preemption trigger $x_{i s}^{*}$ against $K_{e}$.

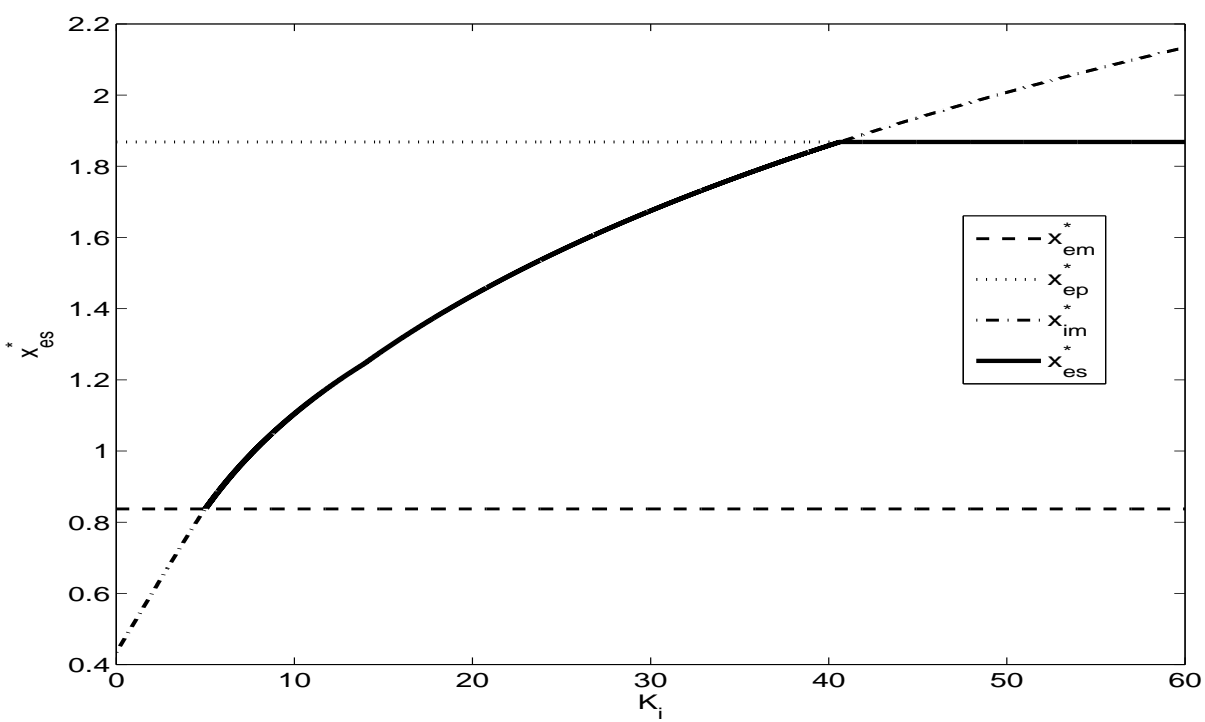

Figure 4: Plot of the entrant's strategic preemption trigger $x_{e s}^{*}$ against $K_{i}$. 


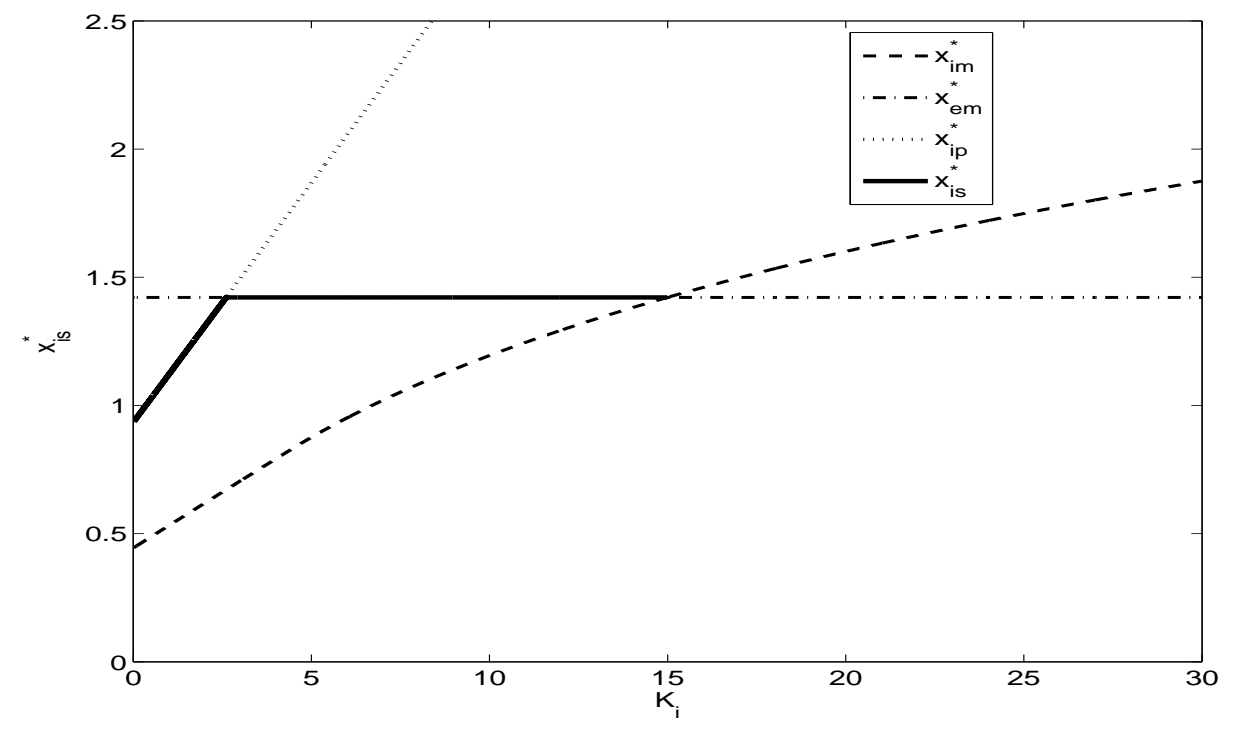

Figure 5: Plot of the incumbent's strategic preemption trigger $x_{i s}^{*}$ against $K_{i}$.

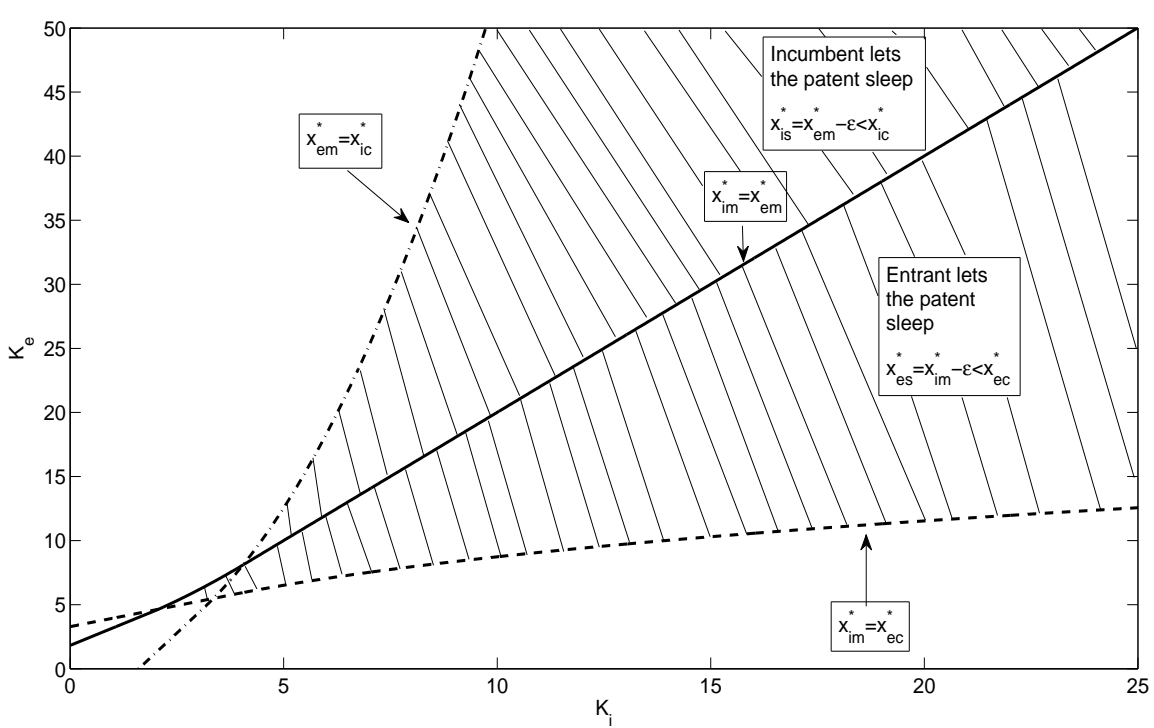

Figure 6: Characterization of the sleeping patent phenomena in the $K_{i}-K_{e}$ plane. 


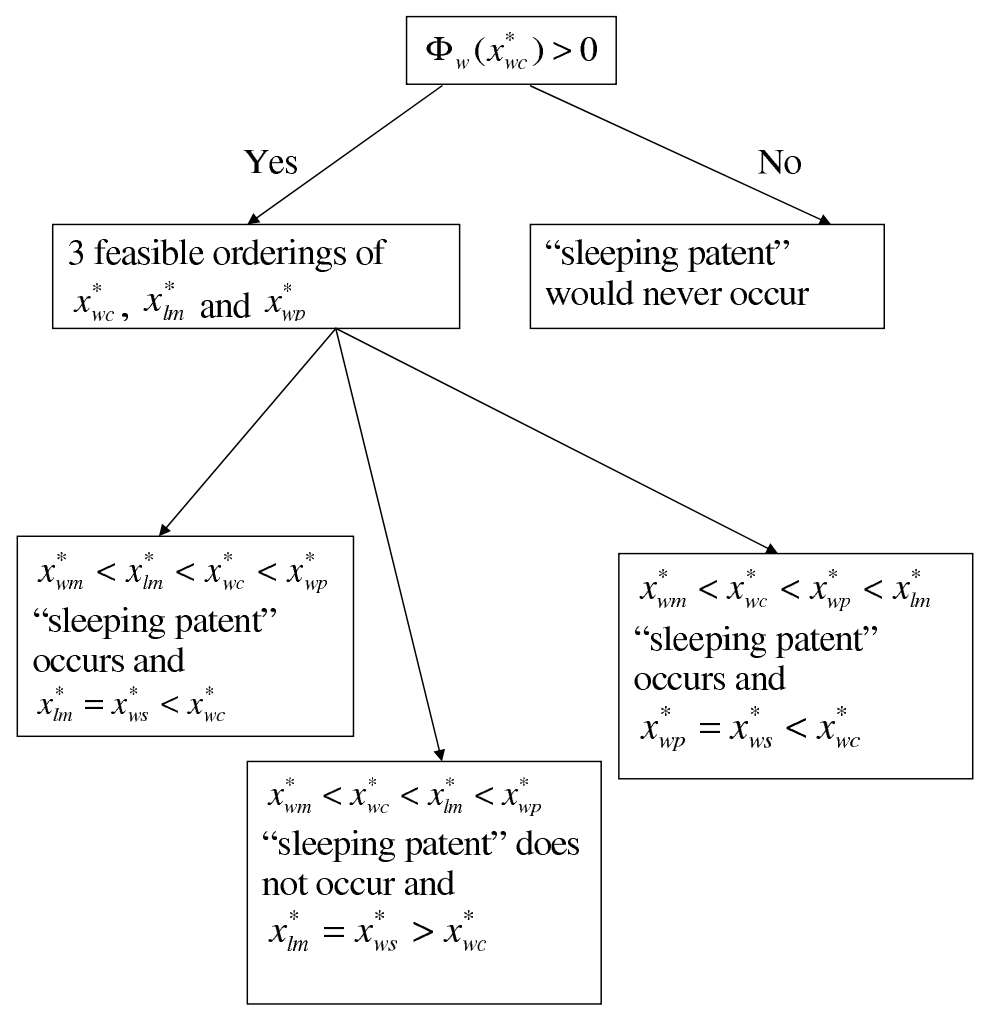

Table 1 Strategies of preemptive patenting adopted by the winner firm. 